\title{
Stability of equilibria of a two-phase Stokes-osmosis problem
}

\author{
FRIEDRICH LIPPOTH \\ Institute of Applied Mathematics, Leibniz University Hannover, Welfengarten 1, \\ 30167 Hannover, Germany \\ E-mail:lippoth@ifam.uni-hannover.de \\ GEORG PROKERT \\ Faculty of Mathematics and Computer Science, TU Eindhoven, P.O. Box 513, \\ 5600 MB Eindhoven, The Netherlands \\ E-mail: g.prokert@tue.nl
}

[Received 17 March 2015]

\begin{abstract}
Within the framework of variational modelling we derive a two-phase moving boundary problem that describes the motion of a semipermeable membrane separating two viscous liquids in a fixed container. The model includes the effects of osmotic pressure and surface tension of the membrane. For this problem we prove that the manifold of steady states is locally exponentially attractive.
\end{abstract}

2010 Mathematics Subject Classification: Primary 35R37; Secondary 35B35, 35K55, 76D07, $76 \mathrm{M} 30$.

Keywords: Variational modelling, two-phase Stokes equations, osmosis, moving boundary problem, maximal $L_{p}$-regularity.

\section{Introduction}

This paper is devoted to a two-phase moving boundary problem describing osmotic swelling of a closed membrane in a viscous liquid.

Let $C \subset \mathbb{R}^{N}(N \geqslant 2)$ be a bounded connected open set with smooth boundary representing a fixed region filled with an incompressible viscous liquid that moves according to the velocity field $u=u(t, x)$. Inside the liquid there is a closed connected semipermeable membrane $\Gamma(t) \subset C$ enclosing an open set $\Omega_{+}(t)$ and separating it from the outer phase $\Omega_{-}(t):=C \backslash \bar{\Omega}_{+}(t)$. In both phases a certain amount of a solute is dissolved. Its scalar concentration $c=c(t, x)$ evolves by convection along $u$ and diffusion through the liquid. It may be discontinuous across $\Gamma(t)$. Both the diffusivities and the viscosities are assumed to be constant and positive but possibly different in $\Omega_{+}$ and $\Omega_{-}$, respectively.

The membrane is permeable for the liquid but impermeable for the solute. Its deformation and movement are governed by surface tension forces, osmotic pressure, and the fluid motion. Based on these assumptions the following moving boundary problem can be derived using the approach of 
variational modelling, see Section 2:

$$
\begin{aligned}
& -v_{ \pm} \Delta u_{ \pm}+\nabla\left(q_{ \pm}+c_{ \pm}\right)=0 \\
& \operatorname{div} u_{ \pm}=0 \\
& \llbracket \tau(u, q+c) \rrbracket n=H n \\
& \llbracket u \rrbracket=0 \\
& u_{-}=0 \\
& \partial_{t} c_{ \pm}-\kappa_{ \pm} \Delta c_{ \pm}+\nabla c_{ \pm} \cdot u_{ \pm}=0 \\
& \kappa_{ \pm} \partial_{n} c_{ \pm}+c_{ \pm}(\llbracket c \rrbracket+H)=0 \\
& \partial_{n} c_{-}=0 \\
& V_{n}=H+\llbracket c \rrbracket+u \cdot n \quad \text { on } \Gamma(t), \quad t>0, \\
& \Gamma(0)=\Gamma^{0}, \\
& c_{ \pm}(0)=c_{ \pm}^{0} \\
& \begin{array}{ll}
\text { in } \Omega_{ \pm}(t), & t>0, \\
\text { in } \Omega_{ \pm}(t), & t>0, \\
\text { on } \Gamma(t), & t>0, \\
\text { on } \Gamma(t), & t>0, \\
\text { on } \partial C, & t>0, \\
& \\
\text { in } \Omega_{ \pm}(t), & t>0, \\
\text { on } \Gamma(t), & t>0, \\
\text { on } \partial C, & t>0, \\
\text { on } \Gamma(t), & t>0, \\
&
\end{array}
\end{aligned}
$$

where we used the notation $u_{ \pm}:=\left.u\right|_{\Omega_{ \pm}}, c_{ \pm}:=\left.c\right|_{\Omega_{ \pm}}$. The brackets $\llbracket \rrbracket$ indicate the jump of a quantity across $\Gamma(t)$, i.e.

$$
\llbracket w(t, \cdot) \rrbracket(x):=\lim _{y \in \Omega_{+}(t), y \rightarrow x} w(t, y)-\lim _{y \in \Omega_{-}(t), y \rightarrow x} w(t, y)
$$

for $x \in \Gamma(t)$ and $w: \Omega_{+}(t) \cup \Omega_{-}(t) \rightarrow \mathbb{R}$. Further, $H=H(t, x)$ denotes the $(N-1)$ - fold mean curvature of the closed compact hypersurface $\Gamma(t)=\partial \Omega_{+}(t)$ at the point $x \in \Gamma(t)$, oriented in the way that spheres have negative curvature, while $V_{n}$ is the normal velocity of the family $\{\Gamma(t)\}$ w.r.t the unit normal field $n=n(t)$ of $\Gamma(t)$ pointing outward $\Omega_{+}(t)$. The operator $\partial_{n}$ takes the directional derivative of a sufficiently regular function w.r.t the normal field $n(t)$. If no confusion seems likely, we use the same symbol $\partial_{n}$ to denote the derivative in the direction normal to $\partial C$ and exterior to $C$ as well. The symbol $q=q(t, x)$ stands for the hydrodynamic pressure and

$$
\tau_{ \pm}\left(u_{ \pm}, q\right):=v^{ \pm} \varepsilon\left(u_{ \pm}\right)-q_{ \pm} \operatorname{Id}:=v^{ \pm}\left(\nabla u_{ \pm}+\left(\nabla u_{ \pm}\right)^{T}\right)-q_{ \pm} \mathrm{Id}, \quad q_{ \pm}:=\left.q\right|_{\Omega_{ \pm}},
$$

is the hydrodynamic stress tensor. Note that the initial velocity $u(0)$ is uniquely determined by $c(0)$ and $\Gamma(0)$ as we shall discuss later in some detail, cf. Section A.1.

System (1.1) is written in dimensionless form. The given positive constants $\kappa_{ \pm}$and $v_{ \pm}$carry information about physical parameters such as diffusivity of the solute, viscosity of the liquid in both phases and permeability of the membrane to solvent. For later use we introduce the piecewise constant functions

$$
v(x, t):=\left\{\begin{array}{ll}
\nu_{+} & x \in \Omega_{+}(t), \\
\nu_{-} & x \in \Omega_{-}(t),
\end{array} \quad \kappa(x, t):= \begin{cases}\kappa_{+} & x \in \Omega_{+}(t), \\
\kappa_{-} & x \in \Omega_{-}(t) .\end{cases}\right.
$$

In the corresponding one-phase situation, a detailed derivation of the model within the framework of variational modelling has been given in [9]. The two-phase problem is obtained in a parallel fashion. Therefore we restrict ourselves here to a brief recapitulation of the chosen setup which will be given in Section 2.

The paper [9] also contains a short-time existence result for classical solutions for the one-phase problem. For the simpler limit problem in which the membrane moves through an immobile liquid 
the existence of classical solutions for a short time has been established in [7], and the paper [8] deals with a stability analysis of its equilibria. For different modelling approaches (excluding fluid motion) as well as analytic results in even more special situations such as radial symmetry we refer to $[11,14,17,18,20,21]$ and the references given in [9].

In this paper we focus on the equilibria of system (1.1) and their stability properties. These equilibria form a finite dimensional submanifold of the phase space. Our main result states that this manifold is locally exponentially attractive, i.e the system is normally stable in the sense of [13].

While the main line of the proof is parallel to the one in [8], we have to deal with the additional difficulty of handling the nonlocal solution operator of the two-phase Stokes system $(1.1)_{1}-(1.1)_{5}$. In particular, the results of [2] that are a crucial ingredient of the stability analysis in $[8,12]$ are no longer directly applicable. Additionally, one has to discuss the full two-phase Stokes system with respect to well-posedness and regularity. As these results do not seem to be readily and explicitly available in the literature, we include a proof of them in an appendix.

The present paper is organized as follows. In Section 2 we explain briefly how our model can be derived within the framework of variational modelling. Section 3 identifies the equilibria of system (1.1). In Section 4 we transform the problem to a fixed reference domain, determine the linearization of the transformed problem around an arbitrary fixed equilibrium and analyse some spectral properties of the corresponding linear operator. In this section we also give a precise formulation of our main result (Theorem 4.2), which is proved in Section 5. The appendix (Section A) contains a detailed discussion of the full two-phase Stokes system (A.1) and some abstract facts that are helpful for the spectral analysis (Lemma 5.1, Corollary 5.2, Lemma 5.3).

\section{Modelling}

We use the same modelling approach as in [9] and derive our model from the following building blocks:

(i) We consider paths in a state manifold $Z$ consisting of pairs $\left(\Omega_{+}, c\right)$ of a simply connected domain $\Omega_{+}$satisfying $\bar{\Omega}_{+} \subset C$ and a nonnegative solute concentration $c: \bar{C} \rightarrow \mathbb{R}$ that may be discontinuous across $\partial \Omega_{+}$. The domain $\Omega_{+}$and the container $C$ uniquely determine $\Omega_{-}$.

(ii) On $\mathcal{Z}$ we define the energy functional

$$
\mathcal{E}\left(\Omega_{+}, c\right):=\gamma \int_{C} c \ln c d x+\alpha\left|\partial \Omega_{+}\right|
$$

with positive constants $\alpha$ and $\gamma$, cf. [9] for a more detailed discussion of their physical meaning. This choice includes diffusion of the solute and surface tension of the membrane as driving mechanisms of the evolution.

(iii) The processes that dissipate energy are solvent motion, solute flux, and passage of solvent through the membrane. Taking into account incompressibility of the solvent and mass conservation of the solute, these processes can be represented by triples

$$
\left\{\left(u, f, V_{n}\right) \mid \operatorname{div} u=0 \text { in } C, \llbracket u \rrbracket=0 \text { on } \partial \Omega_{+}, \quad f_{ \pm} \cdot n=c_{ \pm} V_{n} \text { on } \partial \Omega_{+}\right\}
$$

which we collect in the process space $\mathbb{P}_{\left(\Omega_{+}, c\right)}$.

(iv) The dissipation functional is defined on $\mathbb{P}_{\left(\Omega_{+}, c\right)}$ and given by

$$
\Psi_{\left(\Omega_{+}, c\right)}\left(u, f, V_{n}\right):=\frac{1}{2} \int_{C} \frac{v_{1}|f-c u|^{2}}{c} d x+\frac{1}{2} \int_{C} v_{2}|\varepsilon(u)|^{2} d x+\frac{\nu_{3}}{2} \int_{\partial \Omega_{+}}\left(u \cdot n-V_{n}\right)^{2} d \sigma,
$$


where $v_{j}=v_{j}^{ \pm}$in $\Omega_{ \pm}(j=1,2)$ and $v_{3}$ are positive constants related to mobility of the solute, viscosities of the solvent in both phases and to the membrane's permeability to the solvent, cf. again [9].

(v) Observe that the elements of the tangent spaces $T_{\left(\Omega_{+}, c\right)} \mathcal{Z}$ of the state manifold $\mathcal{Z}$ can be represented by pairs $\left(V_{n}, \dot{c}\right)$, where $V_{n}: \partial \Omega_{+} \longrightarrow \mathbb{R}$ is a normal velocity and $\dot{c}$ is a concentration change. Since mass conservation of the solute is expressed by the relation $\dot{c}+\operatorname{div} f=0$, it seems natural to define the process map $\Pi_{\left(\Omega_{+}, c\right)}: \mathbb{P}_{\left(\Omega_{+}, c\right)} \rightarrow T_{\left(\Omega_{+}, c\right)} \mathcal{Z}$ defined by

$$
\Pi_{\left(\Omega_{+}, c\right)}\left(u, f, V_{n}\right)=\left(V_{n},-\operatorname{div} f\right) .
$$

The model (1.1) is now determined by the dynamical system on $Z$

$$
\dot{z}=\Pi_{z} w^{*}, \quad z=\left(\Omega_{+}, c\right),
$$

where $w^{*}$ is the solution to the minimization problem

$$
\Psi_{z}(w)+\varepsilon^{\prime}(z)\left[\Pi_{z} w\right] \longrightarrow \min , \quad w \in \mathbb{P}_{z},
$$

cf. [9], and by an appropriate scaling.

\section{Equilibria}

Observe that by construction the system (1.1) is a gradient flow w.r.t. the functional

$$
\varepsilon\left(\Omega_{+}, c\right)=\int_{C} c \ln c d x+|\Gamma|
$$

(cf. [9] Section 2 for a more detailed discussion of this fact). Hence, the functional $\varepsilon$ is a Ljapunov function for the system (1.1). Indeed, assuming smoothness and strict positivity of concentrations, integration by parts yields

$$
\frac{d}{d t} \varepsilon\left(\Omega_{+}(t), c(t)\right)=-\int_{C} \frac{|\nabla c(t)|^{2}}{c(t)}-\int_{\Gamma(t)}(\llbracket c(t) \rrbracket+H(t))^{2}-\frac{1}{2} \int_{C}|\varepsilon(u(t))|^{2} .
$$

Let $\left(u, q, c, \Omega_{+}\right)$be an equilibrium solution to (1.1) (i.e. $\left(u, q, c, \Omega_{+}\right)$is constant in time, $\Gamma$ is a closed connected hypersurface, and $u, q, c$ are continuously differentiable away from $\Gamma$ ). Since (3.1) vanishes at equilibria, Korn's inequality implies that $u=0$. Moreover, $c$ must be constant in both phases and $\llbracket c \rrbracket=-H$. Thus, also $H$ is constant, so that $\Gamma$ is a sphere. The first equation in (1.1) implies then that $q$ is constant in both phases, and from the third equation one concludes that $\llbracket q \rrbracket n=0$ on $\Gamma$. Summarizing:

Lemma 3.1 A tupel $\left(u, q, c, \Omega_{+}\right)$is an equilibrium solution to (1.1) iff $\Omega_{+}$is a ball of some radius $R, \bar{\Omega}_{+} \subset C, \llbracket c \rrbracket=(N-1) / R, u=0$ and $q$ is constant in $C$.

\section{Linearization at an equilibrium}

We fix now a single equilibrium $\left(0, \tilde{q}, \tilde{c}, D_{+}\right)$and assume w.l.o.g. that $D_{+}=\mathbb{B}(0,1)$ and $\llbracket \tilde{c} \rrbracket=$ $N-1=: m$. We further define $S:=\partial \mathbb{B}(0,1), D_{-}:=C \backslash \bar{D}_{+}$and keep these notations fixed hereafter. 
In order to solve system (1.1) we are going to consider a set of transformed equations given over $D_{ \pm}$as fixed reference domains. The unknown family of surfaces $\{\Gamma(t)\}$ will be described by a signed distance function with respect to the unit sphere. The ansatz is standard and has already been used in [8] in an identical way.

The mapping

$$
X: S \times(-1,1) \rightarrow \mathbb{R}^{N}, \quad(x, s) \mapsto(1+s) x,
$$

is a smooth diffeomorphism onto its range. Fix $0<a<1$ small enough that $\bar{D} \subset C$, where $D:=\operatorname{range}\left(\left.X\right|_{S \times(a, a)}\right)$. As it is convenient, we decompose the inverse of $X:=\left.X\right|_{S \times(-a, a)}$ into $X^{-1}=(P, \Lambda): D \rightarrow S \times(-a, a)$, where $P$ is the metric projection onto $S$, and $\Lambda$ is the signed distance function with respect to $S$, i.e. $P(x)=x /|x|, \Lambda(x)=|x|-1$. Let $\tilde{a} \in(0, a / 4)$ and

$$
\operatorname{Ad}:=\left\{\sigma \in C^{1}(S) ;\|\sigma\|_{C(S)}<\tilde{a}\right\} .
$$

It is well-known that, given $\sigma \in \mathrm{Ad}$, the mapping $\theta_{\sigma}(x):=(1+\sigma(x)) x$ is a diffeomorphism mapping $S$ onto $S_{\sigma}:=\theta_{\sigma}[S]$. We extend this diffeomorphism to the whole of $\mathbb{R}^{N}$ : Let $\chi \in C^{\infty}(\mathbb{R},[0,1])$ satisfy $\left.\chi\right|_{[-\tilde{a}, \tilde{a}]} \equiv 1,\left.\left.\chi\right|_{(-\infty,-3 \tilde{a}]} \equiv \chi\right|_{[3 \tilde{a}, \infty)} \equiv 0,\left\|\chi^{\prime}\right\|_{\infty}<1 / \tilde{a}$. Then the mapping

$$
y \mapsto \begin{cases}X(P(y), \Lambda(y)+\chi(\Lambda(y)) \cdot \sigma(P(y)), & \text { if } y \in D \\ y, & \text { if } y \notin D\end{cases}
$$

( $\sigma \in \mathrm{Ad}$ ), again denoted by $\theta_{\sigma}$, is an appropriate extension, the so-called Hanzawa diffeomorphism. We have $\theta_{\sigma} \in \operatorname{Diff}\left(\mathbb{R}^{N}, \mathbb{R}^{N}\right)$. Moreover, $\theta_{\sigma} \equiv$ id outside $D$, in particular in a sufficiently small open neighborhood of $\partial C$. Moreover, denoting by $D_{\sigma,+}$ the domain enclosed by $S_{\sigma}$ and letting $D_{\sigma,-}:=C \backslash \bar{D}_{\sigma,+}$, we have that

$$
\left.\theta_{\sigma}\right|_{D_{ \pm}} \in \operatorname{Diff}\left(D_{ \pm}, D_{\sigma, \pm}\right)
$$

$\sigma \in \mathrm{Ad}$, and $\partial D_{\sigma,+}=S_{\sigma}, \partial D_{\sigma,-}=S_{\sigma} \cup \partial C$. Finally note that the surface $S_{\sigma}$ is the zero level set of the function $\varphi_{\sigma}$ defined by

$$
\varphi_{\sigma}(x)=\Lambda(x)-\sigma(P(x)),
$$

$x \in D, \sigma \in$ Ad, i.e. $S_{\sigma}=\varphi_{\sigma}^{-1}[\{0\}]$. For later use we set

$$
L_{\sigma}(x):=\left|\nabla \varphi_{\sigma}\right|\left(\theta_{\sigma}(x)\right) .
$$

It can be shown that $L_{\sigma}>0$ on $S$ for all $\sigma \in$ Ad.

Given $\sigma \in \operatorname{Ad}$, let $\theta_{\sigma}^{*}, \theta_{*}^{\sigma}$ denote the pull-back and push-forward operators induced by $\theta_{\sigma}$, i.e. $\theta_{\sigma}^{*} f=f \circ \theta_{\sigma}, \theta_{*}^{\sigma} g=g \circ \theta_{\sigma}^{-1}$. If the functions $b, \rho$ are time dependent, i.e. $b=b(t, x)$, $\rho=\rho(t, x)$, we define $\left[\theta_{\rho}^{*} b\right](t, x):=\left[\theta_{\rho(t)}^{*} b(t, \cdot)\right](x)$, analogue for $\theta_{*}^{\rho}$.

Using this notation, for $\rho: J \subset[0, \infty) \rightarrow \operatorname{Ad} \cap C^{2}(S)$ and sufficiently smooth $w_{ \pm} \in \mathbb{R}^{D_{ \pm}}$we introduce the transformed operators

$$
\begin{aligned}
n(\rho) & :=\theta_{\rho}^{*} n_{\left[S_{\rho}\right]} ; \\
H(\rho) & :=\theta_{\rho}^{*} H_{\left[S_{\rho}\right]} ; \\
Q_{ \pm}(\rho) w_{ \pm} & :=\theta_{\rho}^{*}\left(\Delta\left(\theta_{*}^{\rho} w_{ \pm}\right)\right) ; \\
\mathbb{B}_{ \pm}(\rho) w_{ \pm} & :=\theta_{\rho}^{*}\left(\left.\nabla\left(\theta_{*}^{\rho} w_{ \pm}\right)\right|_{S_{\rho}}\right) \cdot n(\rho) ; \\
\mathcal{K}_{ \pm}(\rho) w_{ \pm} & :=\theta_{\rho}^{*}\left(\nabla\left(\theta_{*}^{\rho} w_{ \pm}\right)\right) .
\end{aligned}
$$


Letting $\mu_{ \pm}:=c_{ \pm} \circ \theta_{\rho}, \mu_{ \pm, 0}:=c_{ \pm, 0} \circ \theta_{\rho_{0}}$, instead of (1.1), we study the following problem on $D_{ \pm}$as fixed reference domains:

$$
\begin{aligned}
\partial_{t} \mu_{ \pm}-\kappa_{ \pm} Q(\rho) \mu_{ \pm}+\mathcal{K}_{ \pm}(\rho) \mu_{ \pm} \cdot s_{ \pm}(\rho)+R_{ \pm}(\rho, \mu) & =0 & & \text { in } D_{ \pm}, \\
\kappa_{ \pm} \mathbb{B}(\rho) \mu_{ \pm}+\mu_{ \pm}(\llbracket \mu \rrbracket+H(\rho)) & =0 & & \text { on } \partial D_{+}, \\
\partial_{n} \mu \mu_{-} & =0 & & \text { on } \partial C, \\
\partial_{t} \rho-L(\rho)[H(\rho)+\llbracket \mu \rrbracket+s(\rho) \cdot n] & =0 & & \text { on } \partial D_{+}, \\
\mu_{ \pm}(0) & =\mu_{ \pm, 0} & & \text { in } D_{ \pm}, \\
\rho(0) & =\rho_{0}, & &
\end{aligned}
$$

where $s(\rho):=\theta_{\rho}^{*} u$

$$
\begin{aligned}
& -v^{ \pm} \Delta u_{ \pm}+\nabla q_{ \pm}=0 \quad \text { in } D_{\rho, \pm}, \quad t>0, \\
& \operatorname{div} u_{ \pm}=0 \quad \text { in } D_{\rho, \pm}, \quad t>0, \\
& \llbracket \tau(u, q) \rrbracket n=H n \quad \text { on } S_{\rho}, \quad t>0, \\
& \llbracket u \rrbracket=0 \quad \text { on } S_{\rho}, \quad t>0, \\
& u_{-}=0 \quad \text { on } \partial C, \quad t>0 \text {, }
\end{aligned}
$$

and $s_{ \pm}(\rho):=\left.s(\rho)\right|_{D_{ \pm}}$. The terms $\mathbb{R}_{ \pm}$arise from the transformation of the time derivative $\left(\mu_{ \pm}\right)_{t}$ and are determined by

$$
\mathbb{R}_{ \pm}\left(w_{ \pm}, \sigma\right)(y)=r_{0}\left(L_{\sigma}[H(\sigma)+\llbracket w \rrbracket+s(\rho) \cdot n(\rho)], B_{\mu}(\sigma) w_{ \pm}\right)(y), \quad y \in D_{ \pm}
$$

where $w_{ \pm} \in C^{1}\left(\bar{D}_{ \pm}\right), \sigma \in \operatorname{Ad}$ and

$$
\begin{aligned}
& r_{0}(h, k)(y):= \begin{cases}\chi(\Lambda(y)) \cdot h(P(y)) \cdot k(y), & \text { if } \quad y \in D \\
0, & \text { if } y \in \bar{C} \backslash D\end{cases} \\
& B_{\mu}(\sigma) w_{ \pm}(y)=\theta_{\sigma}^{*} \nabla\left(\theta_{*}^{\sigma} w_{ \pm}\right)(y) \cdot\left(n_{S} \circ P\right)(y), \quad y \in D_{ \pm}
\end{aligned}
$$

( $n_{S}$ being the exterior unit normal field of $S$ ). The explicit calculation of $R_{ \pm}$is straightforward, cf. again [3].

Linearization of (4.2) around the equilibrium $\left(\mu_{ \pm}, \rho\right)=\left(\tilde{c}_{ \pm}, 0\right)$ yields the following system for the shifted variable $\mu-\tilde{c}$, denoted again by $\mu$ :

$$
\left.\begin{array}{rlrl}
\partial_{t} \mu_{ \pm}-\kappa_{ \pm} \Delta \mu_{ \pm} & =F_{ \pm}\left(\mu_{ \pm}, \rho\right) & & \text { in } D_{ \pm}, \\
\kappa_{ \pm} \partial_{n} \mu_{ \pm}+\tilde{c}_{ \pm}\left(\llbracket \mu \rrbracket+\left(\Delta_{S}+m\right) \rho\right) & =G_{ \pm}\left(\mu_{ \pm}, \rho\right) & & \text { on } S, \\
\partial_{n} \mu_{-} & =0 & & \text { on } \partial C, \\
\partial_{t} \rho-\left[\left(\Delta_{S}+m\right) \rho+\llbracket \mu \rrbracket+s^{\prime}(0) \rho \cdot n\right] & =\tilde{H}\left(\mu_{ \pm}, \rho\right) & & \text { on } S, \\
\mu_{ \pm}(0) & =\mu_{ \pm, 0} & & \text { in } D_{ \pm}, \\
\rho(0) & =\rho_{0}, & &
\end{array}\right\}
$$

with suitable nonlinear remainders $F, G, H$ that act smoothly between the function spaces we are going to use, cf. Lemma 4.2 in [7] and Corollary A.2 in the present paper. By construction, they satisfy

$$
F_{ \pm}(0)=G_{ \pm}(0)=\tilde{H}(0)=0, \quad F_{ \pm}^{\prime}(0)=G_{ \pm}^{\prime}(0)=\tilde{H}^{\prime}(0)=0
$$


By $\Delta_{S}$ we denote the Laplace-Beltrami operator of the unit sphere. After some algebra, letting $\alpha_{ \pm}:=\kappa_{ \pm} / \tilde{c}_{ \pm}, \tilde{\Delta}:=\Delta_{S}+m$ and

$$
\begin{aligned}
& G_{1}\left(\mu_{ \pm}, \rho\right)=G_{+}\left(\mu_{ \pm}, \rho\right) / \tilde{c}_{+} ; \\
& G_{2}\left(\mu_{ \pm}, \rho\right)=G_{+}\left(\mu_{ \pm}, \rho\right) / \tilde{c}_{+}-G_{-}\left(\mu_{ \pm}, \rho\right) / \tilde{c}_{-} ; \\
& G_{3}\left(\mu_{ \pm}, \rho\right)=\tilde{H}\left(\mu_{ \pm}, \rho\right)+G_{1}\left(\mu_{ \pm}, \rho\right),
\end{aligned}
$$

we get

$$
\begin{aligned}
\partial_{t} \mu_{ \pm}-\kappa_{ \pm} \Delta \mu_{ \pm} & =F_{ \pm}\left(\mu_{ \pm}, \rho\right) & & \text { in } D_{ \pm}, \\
\alpha_{+} \partial_{n} \mu_{+}+\llbracket \mu \rrbracket+\tilde{\Delta} \rho & =G_{1}\left(\mu_{ \pm}, \rho\right) & & \text { on } S, \\
\llbracket \alpha \partial_{n} \mu \rrbracket & =G_{2}\left(\mu_{ \pm}, \rho\right) & & \text { on } S, \\
\partial_{n} \mu- & =0 & & \text { on } \partial C, \\
\partial_{t} \rho+\alpha_{+} \partial_{n} \mu_{+}-s^{\prime}(0) \rho \cdot n & =G_{3}\left(\mu_{ \pm}, \rho\right) & & \text { on } S, \\
\mu_{ \pm}(0) & =\mu_{ \pm, 0} & & \text { in } D_{ \pm}, \\
\rho(0) & =\rho_{0} . & &
\end{aligned}
$$

We close this section by defining the abstract setting for our analysis and giving a precise statement of our main result. Let $p>N+2$. For $s \geqslant 0$ and a Banach space $Y, M \in\left\{D_{ \pm}, S,[0, T],[0, \infty)\right\}$ $(T>0)$ we denote by $W_{p}^{s}(M, Y)$ the $L^{p}$-based Sobolev space of order $s$. In particular, if $s \notin \mathbb{N}$, this fractional-order Sobolev space coincides with the Besov space $B_{p p}^{s}(M, Y)$ (cf. [16]). For the sake of brevity we write $W_{p}^{s}(M):=W_{p}^{s}(M, \mathbb{R})$ and introduce the notations $W_{p}^{s}\left(D_{ \pm}\right):=W_{p}^{s}\left(D_{+}\right) \times$ $W_{p}^{s}\left(D_{-}\right),\left(\mu_{ \pm}, \rho\right):=\left(\mu_{+}, \mu_{-}, \rho\right)$. Let

$$
\begin{aligned}
E_{1} & :=\left\{\left(\mu_{ \pm}, \rho\right) \in W_{p}^{2}\left(D_{ \pm}\right) \times W_{p}^{3-1 / p}(S) ; \partial_{n} \mu_{-}=0 \text { on } \partial C\right\} \\
E & :=\left\{\left(\mu_{ \pm}, \rho\right) \in W_{p}^{2-2 / p}\left(D_{ \pm}\right) \times W_{p}^{3-3 / p}(S) ; \partial_{n} \mu_{-}=0 \text { on } \partial C\right\} \\
E_{0} & :=L^{p}\left(D_{ \pm}\right) \times W_{p}^{1-1 / p}(S)
\end{aligned}
$$

and for an interval $J \subset[0, \infty)$

$$
\mathbb{E}(J):=L_{p}\left(J, E_{1}\right) \cap\left(W_{p}^{1}\left(J, L^{p}\left(D_{ \pm}\right)\right) \times W_{p}^{(3-1 / p) / 2}\left(J, L_{p}(S)\right)\right)
$$

We further define spaces of exponentially decaying functions

$$
\mathbb{E}(\delta):=\left\{\left(\xi_{ \pm}, \sigma\right) \in \mathbb{E}\left(\mathbb{R}^{+}\right) ; e^{\delta t}\left(\xi_{ \pm}, \sigma\right) \in \mathbb{E}\left(\mathbb{R}^{+}\right)\right\}
$$

$(\delta>0)$, equipped with the norm $\left\|\left(\xi_{ \pm}, \sigma\right)\right\|_{\mathbb{E}(\delta)}:=\left\|e^{\delta t}\left(\xi_{ \pm}, \sigma\right)\right\|_{\mathbb{E}\left(\mathbb{R}^{+}\right)}$. and recall the standard embedding result

$$
\mathbb{E}(J) \hookrightarrow C(J, E) .
$$

We formally introduce the operators $\hat{L}, \hat{K}$ and $B$ by their action as follows:

$$
\begin{gathered}
\hat{L}\left(\mu_{ \pm}, \rho\right):=\left(\kappa_{ \pm} \Delta \mu_{ \pm},-\alpha_{+} \partial_{n} \mu_{+}\right), \quad \hat{K}\left(\mu_{ \pm}, \rho\right):=\left(0,0,\left.s^{\prime}(0) \rho\right|_{S} \cdot n\right), \\
B\left(\mu_{ \pm}, \rho\right):=\left(\alpha_{+} \partial_{n} \mu_{+}+\llbracket \mu \rrbracket+\tilde{\Delta} \rho, \llbracket \alpha \partial_{n} \mu \rrbracket\right)
\end{gathered}
$$


where $u=u(\rho)=s^{\prime}(0) \rho$ (and a suitable $p$ ) solve

$$
\begin{aligned}
v_{ \pm} \Delta u_{ \pm}-\nabla p_{ \pm} & =0 & & \text { in } D_{ \pm}, \\
\operatorname{div} u_{ \pm} & =0 & & \text { in } D_{ \pm}, \\
\llbracket \tau(u, p) \rrbracket n & =\tilde{\Delta} \rho n & & \text { on } S, \\
\llbracket u \rrbracket & =0 & & \text { on } S, \\
u_{-} & =0 & & \text { on } \partial C .
\end{aligned}
$$

Then, with $\mathbf{F}:=\left(F_{ \pm}, G_{3}\right), \mathbf{G}:=\left(G_{1}, G_{2}\right),(4.6)$ can be written as an abstract evolution problem

$$
\partial_{t} \mu-(\hat{L}+\hat{K})(\mu)=\mathbf{F}(\mu), \quad B \mu=\mathbf{G}(\mu), \quad \mu(0)=\mu_{0}:=\left(\mu_{ \pm, 0}, \rho_{0}\right), \quad \mu:=\left(\mu_{ \pm}, \rho\right) .
$$

Solutions to (4.9) are paths in the manifold

$$
m:=\{\mu \in E ; B \mu=\mathbf{G}(\mu)\} .
$$

They are supposed to possess the following regularity:

DEFINITION 4.1 A global strong solution of the evolution problem (4.9) is a solution $\mu=\left(\mu_{ \pm}, \rho\right)$ : $[0, \infty) \rightarrow E$ such that

$$
\left.\mu\right|_{[0, T]} \in \mathbb{E}([0, T]) \quad \forall T>0 .
$$

Observe that the set of equilibria of (4.9) is

$$
\varepsilon:=\{\varepsilon \in m ;-(\hat{L}+\hat{K}, B)(\varepsilon)=(\mathbf{F}, \mathbf{G})(\varepsilon)\},
$$

and that these equilibria correspond to the steady states of system (1.1). It is of crucial importance for our analysis that $\varepsilon$ is a submanifold of $m$ of dimension $N+2$, cf. Lemma 2.1 in [8], Proposition 6.4 in [4]. Now we are prepared to state the main theorem of this paper:

Theorem 4.2 There exist $\gamma, \delta>0$ such that, given $\mu_{0} \in \mathbb{B}_{E}(0, \gamma) \cap$ m, problem (4.9) admits a unique global strong solution $\mu=\xi+e$, where $(\xi, e) \in \mathbb{E}(\delta) \times \mathcal{E}$. Moreover, $\mu_{0} \mapsto(\xi, e) \in$ $C^{1}\left(\mathbb{B}_{E}(0, \gamma) \cap m, \mathbb{E}(\delta) \times \varepsilon\right)$.

\section{Spectral analysis and proof of the main result}

In this section we study properties of the operator $L+K: D(L) \subset E_{0} \rightarrow E_{0}$, where $L \mu:=\hat{L} \mu$, $K \mu:=\hat{K} \mu$ and

$$
\begin{aligned}
& D(L):=\left\{\left(\mu_{ \pm}, \rho\right) \in E_{1} ;\right. \\
& \alpha_{+} \partial_{n} \mu_{+}+\llbracket \mu \rrbracket+\tilde{\Delta} \rho=0 \text { on } S, \\
& \llbracket \alpha \partial_{n} \mu \rrbracket=0 \text { on } S, \\
& \partial_{n} \mu_{-}=0\text { on } \partial C .\}
\end{aligned}
$$

We will identify operators and vector spaces with their complexifications without further mentioning.

\section{Lemma 5.1}

(i) The spectrum of $L+K$ consists purely of isolated eigenvalues having eigenspaces of finite dimension.

(ii) The value $\lambda=0$ is an eigenvalue of $L+K$ with $\operatorname{dim} \eta(L+K)=N+2$. 
(iii) All other eigenvalues of $L+K$ are real and negative.

Proof. (i) The operator $L$ generates a strongly continuous analytic semigroup on $E_{0}$ (Theorem 2.2 in [2]). As the operator $\left.\rho \mapsto s^{\prime}(0) \rho\right|_{s} \cdot n$ is of order $1, K$ is a relatively compact perturbation which implies that $L+K$ also generates a strongly continuous analytic semigroup on $E_{0}$ and in particular has a nonempty resolvent set. Since $D(L)$ is compactly embedded in $E_{0}$, the statement follows by [5], Theorem III. 6.29.

(ii) We introduce the bilinear form $\langle\cdot, \cdot\rangle$ by

$$
\left\langle\left(w_{+}, w_{-}, \sigma\right),\left(u_{+}, u_{-}, \theta\right)\right\rangle:=\tilde{c}_{-} \int_{D_{+}} w_{+} u_{+} d x+\tilde{c}_{+} \int_{D_{-}} w_{-} u_{-} d x-\tilde{c}_{+} \tilde{c}_{-} \int_{S} \sigma \tilde{\Delta} \theta d S .
$$

Letting $u(\sigma):=s^{\prime}(0) \sigma$ we observe that

$$
\begin{aligned}
& -\left\langle(L+K)\left(w_{+}, w_{-}, \sigma\right),\left(\bar{w}_{+}, \bar{w}_{-}, \bar{\sigma}\right)\right\rangle \\
& =-\tilde{c}_{-} \kappa_{+} \int_{\Omega_{+}} \Delta w_{+} \bar{w}_{+} d x-\tilde{c}_{+} \kappa_{-} \int_{\Omega_{-}} \Delta w_{-} \bar{w}_{-} d x+\tilde{c}_{+} \tilde{c}_{-} \alpha_{+} \\
& \quad \int_{S} \partial_{n} w_{+}\left(\alpha_{+} \partial_{n} \bar{w}_{+}+\llbracket \bar{w} \rrbracket\right) d S+\int_{S} u(\sigma) \cdot n \tilde{\Delta} \bar{\sigma} d S \\
& =\tilde{c}_{-} \kappa_{+}\left\|\nabla w_{+}\right\|_{L^{2}\left(\Omega_{+}\right)}^{2}+\tilde{c}_{+} \kappa_{-}\left\|\nabla w_{-}\right\|_{L^{2}\left(\Omega_{-}\right)}^{2}+\kappa_{+} \alpha_{+} \tilde{c}_{-}\left\|\partial_{n} w_{+}\right\|_{L^{2}(S)}^{2} \\
& \quad+\frac{1}{2}\left(\int_{D_{+}}|\varepsilon(u(\sigma))|^{2} d x+\int_{D_{-}}|\varepsilon(u(\sigma))|^{2} d x\right) \geqslant 0
\end{aligned}
$$

for all $\left(w_{+}, w_{-}, \sigma\right) \in D(L)$. Suppose $(L+K)\left(w_{+}, w_{-}, \sigma\right)=0$ for $\left(w_{+}, w_{-}, \sigma\right) \in D(L)$. Then (5.1) implies that $w_{ \pm}$are constant on $D_{ \pm}$(thus $\llbracket w \rrbracket$ is constant on $S$ ) and that $u(\sigma)=0$ in $C$. From elementary properties of $\Delta_{S}$ and the results from Sections A.1.1, A.1.3 we hence get that

$$
\eta(L+K)=\operatorname{span}\left\{(m, 0,-1),(0, m, 1),\left(0,0, x_{1}\right), \ldots,\left(0,0, x_{N}\right)\right\}=:\left\{\varepsilon_{1}, \ldots, \varepsilon_{N+2}\right\} .
$$

(iii) The computation (5.1) shows that $-\left\langle(L+K)\left(w_{+}, w_{-}, \sigma\right),\left(\bar{w}_{+}, \bar{w}_{-}, \bar{\sigma}\right)\right\rangle \geqslant 0$ for all $\left(w_{+}, w_{-}, \sigma\right) \in D(L)$. Using the fact that $\int_{S} u(\sigma) \cdot n=0$, we can show in completely the same fashion as in the proof of Lemma 3.1 iii) in [8] that $\left\langle\left(w_{+}, w_{-}, \sigma\right),\left(\bar{w}_{+}, \bar{w}_{-}, \bar{\sigma}\right)\right\rangle \geqslant 0$ for all eigenvectors $\left(w_{+}, w_{-}, \sigma\right)$ of $L+K$ with equality only if $\left(w_{+}, w_{-}, \sigma\right) \in \eta(L+K)$. Hence, the assertion follows from Lemma A.4 and (i).

Corollary 5.2 We have

$$
\sup \operatorname{Re}(\sigma(L+K) \backslash\{0\})<0 .
$$

Lemma 5.3 The zero eigenvalue of $L+K$ is semisimple, i.e. $X=\eta(L+K) \oplus \mathbb{R}(L+K)$.

Proof. As $L+K$ has nonempty resolvent set and $D(L)$ is compactly embedded in $E_{0}, L+K$ (considered as a bounded operator from $D(L)$ to $E_{0}$ ) is Fredholm and has index zero. Hence, by Corollary A.6, it suffices to show that $\eta(L+K) \cap \mathbb{R}(L+K)=\{0\}$. We introduce the linear mapping

$$
\Phi\left(f_{+}, f_{-}, \theta\right):=\left(\tilde{c}_{+} \int_{S} \theta d S+\int_{D_{+}} f_{+} d x, \tilde{c}_{-} \int_{S} \theta d S-\int_{D_{-}} f_{-} d x\right)
$$


and verify assumptions (i), (ii) and (iii) of Lemma A.3 with

$$
\left(\left(w_{+}, w_{-}, \theta\right) \mid\left(v_{+}, v_{-}, \tau\right)\right)=\left\langle\left(w_{+}, w_{-}, \theta\right),\left(\bar{v}_{+}, \bar{v}_{-}, \bar{\tau}\right)\right\rangle
$$

and $V=\mathbb{C}^{2}$. Observe that by (5.1) (and the considerations below (5.1)) we have that

$$
\left\langle(L+K)\left(w_{+}, w_{-}, \sigma\right),\left(\bar{w}_{+}, \bar{w}_{-}, \bar{\sigma}\right)\right\rangle=0 \text { iff }\left(\bar{w}_{+}, \bar{w}_{-}, \bar{\sigma}\right) \in \eta_{(L+K)}
$$

The divergence theorem and the fact that $\int_{S} u(\theta) \cdot n d S=0$ imply that $\Phi$ vanishes on $\mathbb{R}(L+K)$. Assume that $z:=\left(w_{+}, w_{-}, \sigma\right) \in \eta(L+K) \cap \eta(\Phi),\left(w_{+}, w_{-}, \sigma\right)=\sum_{j=1}^{N+2} \alpha_{j} \varepsilon_{j}$ (cf. 5.2). Then, as $\int_{S} x_{j} d S=0$ (using $\alpha_{ \pm}=\kappa_{ \pm} / \tilde{c}_{ \pm}$), $\Phi z=0$ means that

$$
\int_{S}\left(\alpha_{2}-\alpha_{1}\right) d S=-\int_{D_{+}} \frac{\alpha_{1} m}{\tilde{c}_{+}} d S
$$

and

$$
\int_{S}\left(\alpha_{2}-\alpha_{1}\right) d S=+\int_{D_{-}} \frac{\alpha_{2} m}{\tilde{c}_{-}} d S
$$

This is equivalent to $A\left(\alpha_{1}, \alpha_{2}\right)=0$, where

$$
A=\left(\begin{array}{cc}
-m\left|D_{+}\right| / \tilde{c}_{+}+|S| & -|S| \\
+|S| & m\left|D_{-}\right| / \tilde{c}_{-}-|S|
\end{array}\right) .
$$

We calculate, using $|S|=N\left|D_{+}\right|, \llbracket \tilde{c} \rrbracket=m=N-1$

$$
\operatorname{det}(A)=\frac{-m|S|\left|D_{-}\right|}{N \tilde{c}_{-}}+\frac{m|S|\left|D_{-}\right|}{\tilde{c}_{-}}+\frac{m|S|\left|D_{+}\right|}{\tilde{c}_{+}}+\frac{m\left|D_{+}\right|\left|D_{-}\right|}{\tilde{c}_{+}}>0,
$$

i.e. $\alpha_{1}=\alpha_{2}=0$. Hence, $z \in \mathcal{O}$.

We turn to the proof of Theorem 4.2. Lemmas $5.1-5.3$ allow to follow the same strategy as in [8], Section 4. In fact, the arguments given there can literally be repeated here if one only replaces the operators $\hat{L}, L$ by the operators $\hat{L}+\hat{K}, L+K$, respectively. However, the proof of [8], Lemma 4.3 has to be modified because Theorem 2.2 in [2] does not apply to the nonlocal operator $L+K$. Nevertheless, the analogous result holds true:

Lemma 5.4 Let $\omega>0$. Then

$$
(\omega-(\hat{L}+\hat{K}), B) \in \mathcal{L}_{\text {is }}\left(E, W_{p}^{-2 / p}\left(D_{ \pm}\right) \times\left[W_{p}^{1-3 / p}(S)\right]^{3}\right) \cap \mathcal{L}_{\text {is }}\left(E_{1}, L_{p}\left(D_{ \pm}\right) \times\left[W_{p}^{1-1 / p}(S)\right]^{3}\right) .
$$

In accordance with our general notation, we denote by $W_{p}^{-2 / p}\left(D_{ \pm}\right):=B_{p p}^{-2 / p}\left(D_{ \pm}\right)$a Besov space of negative differentiability order, see [16].

Proof. From Lemma 4.3 in [8] we know that

$$
(\omega-\hat{L}, B) \in \mathcal{L}_{\mathrm{is}}\left(E, W_{p}^{-2 / p}\left(D_{ \pm}\right) \times\left[W_{p}^{1-3 / p}(S)\right]^{3}\right) \cap \mathcal{L}_{\mathrm{is}}\left(E_{1}, L_{p}\left(D_{ \pm}\right) \times\left[W_{p}^{1-1 / p}(S)\right]^{3}\right) .
$$

In particular, $(\omega-\hat{L}, B)$ is Fredholm and has index 0 . Since $\hat{K}$ is a compact perturbation, the same is true for the operator $(\omega-(\hat{L}+\hat{K}), B)$. Therefore it suffices to show that $(\omega-(\hat{L}+\hat{K}), B)$ is injective. 
We first consider this operator as an element of $\mathcal{L}\left(E_{1}, L_{p}\left(D_{ \pm}\right) \times\left[W_{p}^{1-1 / p}(S)\right]^{3}\right)$. Then, injectivity is a direct consequence of Lemma 5.1 .

To prove the remaining part, assume

$$
(\omega-(\hat{L}+\hat{K}), B) \mu=0, \quad \mu=\left(\mu_{ \pm}, \rho\right) \in E,
$$

or equivalently

$$
(\omega-\hat{L}, B) \mu=\left(0,0,\left.s^{\prime}(0) \rho\right|_{S} \cdot n, 0,0\right) .
$$

Recall that $s^{\prime}(0) \rho$ is defined by the BVP (4.8). Applying Theorem A.1 to this problem and using $\rho \in W_{p}^{3-3 / p}(S), p>2$, we find that the right side of (5.4) is in $L_{p}\left(D_{ \pm}\right) \times\left[W_{p}^{1-1 / p}(S)\right]^{3}$, so (5.3) yields $\mu \in E_{1}$. The result follows again by Lemma 5.1.

\section{Conclusion}

Our analysis crucially relies on the fact that the problem under consideration belongs to the class of parabolic evolutions, in the general sense that the semigroup of operators (on appropriate function spaces) arising as solution of the linearized evolution problem is analytic. Corresponding maximal regularity results allow the treatment of the nonlinearities introduced by the transformation to a fixed domain. As typical for the techniques used here, they provide smooth solutions but are (in absence of further structural information) restricted to "perturbative" results, producing either shorttime solutions (as in [9] for the present problem) or long-time solutions near equilibria or periodic solutions.

The present paper shows that these techniques are strong and versatile enough to treat relatively complex models in which coupled evolutions in two phases and on their interface as well as additional elliptic systems occur. On a technical level, this is reflected in the fact that we use products of spaces of functions with different domains of definitions and a solution operator for the Stokes equations. In a sense, using this solution operator allows to treat the present problem as a perturbed version of the problem without flow, with the perturbation being "of lower order."

The convergence result may be viewed as an application of a suitably generalized principle of linearized stability to a nonlinear parabolic problem, which is also well established by now. Discussing the spectrum of the linearization at an equilibrium provides additional structural information to conclude that a solution starting close to the manifold of equilibria is actually global and converges to this manifold at an exponential rate.

In this respect, it remains an open and interesting question whether, and how, structural properties like parabolicity and stability of equilibria can be concluded already from properties of the initial ingredients of the variational model, and not only from the resulting moving boundary problem.

\section{A. Appendix}

\section{A.1 Two-phase Stokes equations}

Let $C \subset \mathbb{R}^{N}$ be the set defined in the introduction. In this section we denote by $\Omega_{+}$a bounded simply connected open set with boundary $\partial \Omega_{+}$of class $C^{\infty}$ such that $\bar{\Omega}_{+} \subset C$ and define $\Omega_{-}:=$ $C \backslash \bar{\Omega}_{+}$. Moreover, $n$ denotes the outward unit normal field of $\partial \Omega_{+}$. If no confusion seems likely, 
the symbol $\partial_{n}$ stands for both the directional derivative w.r.t. $n$ and w.r.t. the outer unit normal field of $\partial C$. We are interested in the two-phase Stokes system

$$
\begin{aligned}
-v_{ \pm} \Delta u_{ \pm}+\nabla p_{ \pm} & =f_{ \pm} & & \text {in } \Omega_{ \pm}, \\
-\operatorname{div} u_{ \pm} & =g_{ \pm} & & \text {in } \Omega_{ \pm}, \\
\llbracket \tau(u, p) \rrbracket n & =h & & \text { on } \partial \Omega_{+}, \\
\llbracket u \rrbracket & =l & & \text { on } \partial \Omega_{+}, \\
u_{-} & =0 & & \text { on } \partial C
\end{aligned}
$$

and consider first the question of unique solvability of the simplified problem

$$
\begin{aligned}
-v_{ \pm} \Delta u_{ \pm}+\nabla p_{ \pm} & =f_{ \pm} & & \text {in } \Omega_{ \pm}, \\
-\operatorname{div} u_{ \pm} & =g_{ \pm} & & \text {in } \Omega_{ \pm}, \\
\llbracket \tau(u, p) \rrbracket n & =h & & \text { on } \partial \Omega_{+}, \\
\llbracket u \rrbracket & =0 & & \text { on } \partial \Omega_{+}, \\
u_{-} & =0 & & \text { on } \partial C .
\end{aligned}
$$

A.1.1 Weak solutions. Let $H:=H_{0}^{1}\left(C, \mathbb{R}^{N}\right)=\left\{w \in W_{2}^{1}\left(C, \mathbb{R}^{N}\right) ; w=0\right.$ on $\left.\partial C\right\}$, $Q:=L_{2}(C)$,

$$
a:=H \times H \rightarrow \mathbb{R}, \quad(u, \varphi) \mapsto \int_{C} v(\varepsilon(u): \varepsilon(\varphi)),
$$

$v=v_{ \pm}$in $\Omega_{ \pm}$, and

$$
b:=Q \times H \rightarrow \mathbb{R}, \quad(q, \varphi) \mapsto-\int_{C} q \operatorname{div} \varphi .
$$

A weak solution of the system A.2 $\left((f, g): C \rightarrow \mathbb{R}^{N+1}, \quad h: \partial \Omega_{+} \rightarrow \mathbb{R}^{N}\right)$ is a pair $(u,[q]) \in H \times Q / \sim_{c}\left(f \sim_{c} g: \Leftrightarrow f=g+\right.$ const $)$ that satisfies

$$
a(u, \varphi)+b(q, \varphi)=\int_{\partial \Omega_{+}} h \varphi+\int_{C} f \varphi+\int_{C} \nabla g \varphi \quad \text { for all } \varphi \in H \quad(q \in[q])
$$

as well as

$$
b(\psi, u)=g \quad \text { for all } \psi \in Q .
$$

Due to Korn's inequality, the bilinear form $a$ is coercive on $H$. Moreover, the bilinear form $b$ induces a linear operator

$$
B: H \rightarrow Q^{\prime}, \quad B u(\psi):=b(\psi, u) .
$$

If $Q$ is identified with its dual by means of the Riesz isomorphism, the range of $B$ is the set $\left\{r \in L_{2}(C) ; \int_{C} r=0\right\}$. Since this is a closed subset of $L_{2}(C)$, classical results (cf. [1], Section II.1) imply that there is a unique weak solution of (A.2) for every $(f, g, h) \in L^{2}\left(C, \mathbb{R}^{N}\right) \times H^{1}(C, \mathbb{R}) \times L_{2}\left(\partial \Omega_{+}, \mathbb{R}^{N}\right)$, provided $\int_{C} g=0$.

A.1.2 The Lopatinskii-Shapiro condition. We want to show that the two-phase Stokes system (A.1) satisfies the Lopatinskii-Shapiro condition. W.1.o.g. we restrict ourselves to the halfspace situation, i.e., the case $\Omega_{ \pm}:=\mathbb{R}^{N-1} \times \mathbb{R}^{ \pm}$. 
By reflecting $u_{-}$in system (A.1) to the upper half space, the operator on the left hand side of system (A.1) can be expressed by the $(2 N+2) \times(2 N+2)$ and the $(2 N) \times(2 N+2)$ matrices of operators

$$
A\left(\partial_{1}, \ldots, \partial_{N}\right):=\left(\begin{array}{ccccc}
-v_{+} \boldsymbol{\Delta} \nabla & 0 & 0 \\
\nabla^{T} & 0 & 0 & 0 \\
0 & 0 & -\nu_{-} \boldsymbol{\Delta} & \tilde{\nabla} \\
0 & 0 & \tilde{\nabla}^{T} & 0
\end{array}\right), \quad B\left(\partial_{1}, \ldots, \partial_{N}\right):=\left(\begin{array}{cccc}
\mathbf{B}^{+} & -\mathbf{e}_{\mathbf{N}} & -\mathbf{B}^{-} & \mathbf{e}_{\mathbf{N}} \\
\mathbf{I} & \mathbf{0} & -\mathbf{I} & \mathbf{0}
\end{array}\right)
$$

both acting on vectors $\left(u_{+}, p_{+}, u_{-}, p_{-}\right)^{T}$, where $u_{ \pm}:=\left(u_{ \pm}^{1}, \ldots, u_{ \pm}^{N}\right)$. Here, we used the $N \times N$ matrices of operators

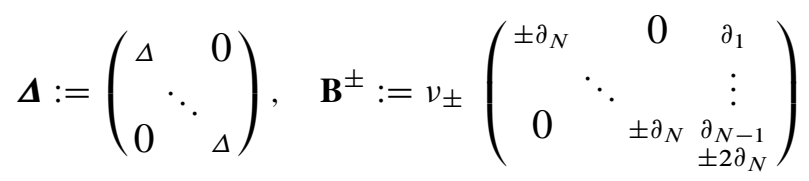

and the notation $\tilde{\nabla}:=\left(\partial_{1}, \ldots, \partial_{N-1},-\partial_{N}\right)^{T}, \mathbf{e}_{\mathbf{N}}:=(0, \ldots, 0,1) \in \mathbb{R}^{N}$. The operator $A$ represents a Douglis-Nirenberg elliptic system (cf. [19]) with DN-numbers

$$
\begin{gathered}
s_{1}=\ldots=s_{N}=s_{N+2}=\ldots=s_{2 N+1}=t_{1}=\ldots=t_{N}=t_{N+2}=\ldots=t_{2 N+1}=1, \\
s_{N+1}=s_{2 N+2}=t_{N+1}=t_{2 N+2}=0
\end{gathered}
$$

and it coincides with its principal part (note that $\sum s_{j}+t_{j}=4 N=\operatorname{ord}(A)$ ). The characteristic polynomial is $v_{+}^{N-1} v_{-}^{N-1}\left(|\xi|^{2}+\lambda^{2}\right)^{2 N}$, where $\xi=\left(\xi_{1}, \ldots, \xi_{N-1}\right) \in \mathbb{R}^{N-1}$ and $\lambda \in \mathbb{R}$.

We have to determine a $2 N$-dimensional space $m^{0}$ of exponentially decaying solutions to the initial value problem $A\left(i \xi_{1}, \ldots, i \xi_{N-1}, \partial_{t}\right)\left(u_{+}, p_{+}, u_{-}, p_{-}\right)=0$, i.e. (letting $\left.v_{ \pm}:=u_{ \pm}^{N}\right)$

$$
\left\{\begin{aligned}
v_{ \pm}\left(|\xi|^{2}-\partial_{t}^{2}\right) u_{ \pm}^{j} & =-i \xi_{j} p_{ \pm} \quad j=1, \ldots, N-1 \\
v_{ \pm}\left(|\xi|^{2}-\partial_{t}^{2}\right) v_{ \pm} & =\mp \partial p_{ \pm} \\
\sum_{j=1}^{N-1} \xi_{j} u_{ \pm}^{j} & = \pm i \partial v_{ \pm}
\end{aligned}\right.
$$

This system can be solved to the result

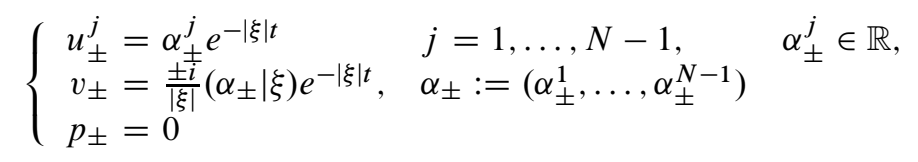

and

$$
\left\{\begin{aligned}
\tilde{u}_{ \pm}^{j} & =\beta_{ \pm} i\left(\frac{\xi_{j}}{|\xi|^{2}}-\frac{t \xi_{j}}{|\xi|}\right) e^{-|\xi| t}, & & j=1, \ldots, N-1 \\
\tilde{v}_{ \pm} & = \pm \beta_{ \pm} t e^{-|\xi| t} & & \\
\tilde{p}_{ \pm} & =2 \beta_{ \pm} v_{ \pm} e^{-|\xi| t}, & & \beta_{ \pm} \in \mathbb{R}
\end{aligned}\right.
$$

To verify the Lopatinskii-Shapiro condition we have to check that the problem

$$
\left.B\left(i \xi_{1}, \ldots, i \xi_{N-1}, \partial_{t}\right)\left(u_{+}, p_{+}, u_{-}, p_{-}\right)\right|_{t=0}=0, \quad\left(u_{+}, p_{+}, u_{-}, p_{-}\right) \in m^{0},
$$

possesses no nontrivial solutions. Assuming (A.6) and writing simply

$$
\left(u_{ \pm}^{1}, \ldots, u_{ \pm}^{N-1}, v_{ \pm}\right) \text {instead of }\left(u_{ \pm}^{1}, \ldots, u_{ \pm}^{N-1}, v_{ \pm}\right)+\left(\tilde{u}_{ \pm}^{1}, \ldots, \tilde{u}_{ \pm}^{N-1}, \tilde{v}_{ \pm}\right)
$$


we first use

$$
\left[\left(u_{+}^{1}, \ldots, u_{+}^{N-1}, v_{+}\right)-\left(u_{-}^{1}, \ldots, u_{-}^{N-1}, v_{-}\right)\right](0)=0
$$

This implies that

$$
\alpha_{+}^{j}-\alpha_{-}^{j}+\frac{i \xi_{j}}{|\xi|^{2}}\left(\beta_{+}-\beta_{-}\right)=0, \quad j=1, \ldots, N-1, \quad\left(\alpha_{+} \mid \xi\right)=-\left(\alpha_{-} \mid \xi\right) .
$$

Multiplication by $\xi$ yields

$$
\left(\alpha_{ \pm} \mid \xi\right)=\frac{\mp i}{2}\left(\beta_{+}-\beta_{-}\right)
$$

Further, (A.6) implies

$$
\left[v_{+}\left[\left(\begin{array}{c}
\partial_{t} u_{+}^{1} \\
\vdots \\
\partial_{t} u_{+}^{N-1} \\
\partial_{t} v_{+}
\end{array}\right)+\left(\begin{array}{c}
i \xi_{1} v_{+} \\
\vdots \\
i \xi_{N-1} v_{+} \\
\partial_{t} v_{+}
\end{array}\right)\right]-v_{-}\left[\left(\begin{array}{c}
-\partial_{t} u_{-}^{1} \\
\vdots \\
-\partial_{t} u_{-}^{N-1} \\
-\partial_{t} v_{-}
\end{array}\right)+\left(\begin{array}{c}
i \xi_{1} v_{-} \\
\vdots \\
i \xi_{N-1} v_{-} \\
-\partial_{t} v_{-}
\end{array}\right)\right]-\left(\begin{array}{c}
0 \\
\vdots \\
0 \\
p_{+}-p_{-}
\end{array}\right)\right](0)
$$

so we find that

$$
\begin{aligned}
& v_{+} {\left[\left(\begin{array}{c}
-\alpha_{+}|\xi| \\
-i\left(\alpha_{+} \mid \xi\right)
\end{array}\right)+\left(\begin{array}{c}
-\xi\left(\alpha_{+} \mid \xi\right) /|\xi| \\
-i\left(\alpha_{+} \mid \xi\right)
\end{array}\right)+\left(\begin{array}{c}
-2 i \beta_{+} \xi /|\xi| \\
\beta_{+}
\end{array}\right)+\left(\begin{array}{c}
0 \\
\beta_{+}
\end{array}\right)\right] } \\
&\left.-v_{-}\left[\left(\begin{array}{c}
\alpha-|\xi| \\
-i\left(\alpha_{-} \mid \xi\right)
\end{array}\right)+\left(\begin{array}{c}
\xi\left(\alpha_{-} \mid \xi\right) /|\xi| \\
-i\left(\alpha_{-} \mid \xi\right)
\end{array}\right)+\left(\begin{array}{c}
2 i \beta_{-} \xi /|\xi| \\
\beta_{-}
\end{array}\right)+\left(\begin{array}{c}
0 \\
\beta_{-}
\end{array}\right)\right]-\left[\begin{array}{c}
0 \\
v_{+} \\
2 \beta_{+}
\end{array}\right)-v_{-}\left(\begin{array}{c}
0 \\
2 \beta_{-}
\end{array}\right)\right] \\
&=0 .
\end{aligned}
$$

By multiplying the first row in (A.10) by $\xi$ and using (A.8) this gives the linear system

$$
M \vec{\beta}:=\left(\begin{array}{cc}
v_{+}+v_{-} & v_{+}+v_{-} \\
v_{+}+v_{-} & -\left(v_{+}+v_{-}\right)
\end{array}\right)\left(\begin{array}{c}
\beta_{+} \\
\beta_{-}
\end{array}\right)=0
$$

which possesses only the trivial solution $\beta_{+}=\beta_{-}=0$ since $\operatorname{det}(M)=-2\left(v_{+}+v_{-}\right)^{2}<0$. Hence, by (A.7), (A.8) $\alpha_{+}=\alpha_{-}$and $\left(\alpha_{+} \mid \xi\right)=\left(\alpha_{-} \mid \xi\right)=0$. Using this, the first row in (A.10) reduces to

$$
\left(v_{+}+v_{-}\right) i|\xi| \alpha_{+}=0
$$

and hence also $\alpha_{+}=\alpha_{-}=0$. Therefore, the Lopatinskii-Shapiro condition is satisfied.

A.1.3 Regularity. We are now interested in strong/classical solutions of the system (A.1) under the necessary solvability demand

$$
\int_{\Omega_{+}} g_{+}+\int_{\Omega_{-}} g_{-}=-\int_{\partial \Omega_{+}} l \cdot n
$$

Let

$$
\begin{aligned}
& \Lambda_{v_{+}, v_{-}}\left(u_{+}, p_{+}, u_{-}, p_{-}\right):= \\
& \left(v_{+} \Delta u_{+}-\nabla p_{+}, v_{-} \Delta u_{-}-\nabla p_{-},-\operatorname{div} u_{+},-\operatorname{div} u_{-}, \llbracket \tau(u, p) \rrbracket n, \llbracket u \rrbracket\right) .
\end{aligned}
$$


From Theorem 9.32 in [19] and Section A.1.2 we know that the operators $\Lambda_{v_{+}, v_{-}}$, considered as bounded operator between appropriate function spaces (see Theorem A.1 below), are Fredholm for all positive $v_{+}, v_{-}$. In order to calculate their index, we first consider the case that $v_{+}=v_{-}=: v>0$ and determine the range of $\Lambda_{v, v}$ (for the sake of brevity we refrain from stating regularities as they can be easily added by means of classical elliptic theory and the results from [6], Section $3.3,3.5)$ : let $l_{+}, l_{-}$satisfy

- $l_{+}-l_{-}=l$ on $\partial \Omega_{+}$;

- $\int_{\Omega_{ \pm}} g_{ \pm}=\mp \int_{\partial \Omega_{+}} l_{ \pm} \cdot n$.

Taking into account (A.12), one possible choice is $l_{+}:=l+\left.l_{-}\right|_{\partial \Omega_{+}}$, where $l_{-}:=\nabla L$ and $L$ solves

$$
\begin{aligned}
-\Delta L & =g_{-} & & \text {in } \Omega_{-}, \\
\partial_{n} L & =\frac{\int_{\Omega_{-}} g_{-}}{\partial \Omega_{+}} & & \text {on } \partial \Omega_{+}, \\
\partial_{n} L & =0 & & \text { on } \partial C .
\end{aligned}
$$

Further, let $w_{ \pm}:=\nabla W_{ \pm}$, where $W_{ \pm}$solve

$$
\begin{aligned}
& -\Delta W_{+}=g_{+} \quad \text { in } \Omega_{+}, \\
& \partial_{n} W_{+}=l_{+} \cdot n \quad \text { on } \partial \Omega_{+} \text {, } \\
& -\Delta W_{-}=g_{-} \quad \text { in } \Omega_{-} \text {, } \\
& \partial_{n} W_{-}=l_{-} \cdot n \quad \text { on } \partial \Omega_{+} \text {, } \\
& \partial_{n} W_{-}=0 \quad \text { on } \partial C \text {. }
\end{aligned}
$$

Note that $-\operatorname{div} w_{ \pm}=g_{ \pm}$in $\Omega_{ \pm}$. We extend $f_{-}$and $\Delta w_{-}$to $\mathbb{R}^{N} \backslash \bar{\Omega}_{+}$in such a way that they vanish outside some open ball containing $\bar{C}$ and consider the problems

$$
\begin{aligned}
-v \Delta v_{+}+\nabla q_{+} & =f_{+}+v \Delta w_{+} & & \text {in } \Omega_{+}, \\
\operatorname{div} v_{+} & =0 & & \text { in } \Omega_{+}, \\
v_{+} & =l_{+}-w_{+} & & \text {on } \partial \Omega_{+}, \\
-v \Delta v_{-}+\nabla q_{-} & =f_{-}+v \Delta w_{-} & & \text {in } \mathbb{R}^{N} \backslash \bar{\Omega}_{+}, \\
\operatorname{div} v_{-} & =0 & & \text { in } \mathbb{R}^{N} \backslash \overline{\Omega_{+}}, \\
v_{-} & =l_{-}-w_{-} & & \text {on } \partial \Omega_{+} .
\end{aligned}
$$

It follows from Section 3.5 in [6] that the problems (A.17) and (A.18) possess classical solutions (since $w_{ \pm} \cdot n=l_{ \pm} \cdot n$ on $\partial \Omega_{ \pm}$). Moreover, $\int_{\partial C} v_{-} \cdot n_{\partial C}=0$. Next we are interested in the system

$$
\begin{aligned}
-v \Delta u_{+}+\nabla p_{+} & =0 & & \text { in } \Omega_{+}, \\
-v \Delta u_{-}+\nabla p_{-} & =0 & & \text { in } \mathbb{R}^{N} \backslash \bar{\Omega}_{+}, \\
\operatorname{div} u_{+} & =0 & & \text { in } \Omega_{+}, \\
\operatorname{div} u_{-} & =0 & & \text { in } \mathbb{R}^{N} \backslash \overline{\Omega_{+}}, \\
\llbracket \tau(u, p) \rrbracket n & =h-\llbracket \tau(w+v, q) \rrbracket n & & \text { on } \partial \Omega_{+}, \\
\llbracket u \rrbracket & =0 & & \text { on } \partial \Omega_{+},
\end{aligned}
$$

where $v, w: \Omega_{+} \cup \Omega_{-} \rightarrow \mathbb{R}^{N}, q: \Omega_{+} \cup \Omega_{-} \rightarrow \mathbb{R}$ are defined in the obvious way. The single layer potential with density $\psi$ and w.r.t. the constant viscosity $v>0$ is given by

$$
\begin{aligned}
& V(x, \psi):=\frac{1}{2 v \omega_{N}} \int_{\Gamma}\left(\frac{1}{(n-2)|x-y|^{N-2}}+\frac{(x-y)(x-y)^{T}}{|x-y|^{N}}\right) \psi(y) d \sigma(y) \\
& Q(x, \psi):=\frac{1}{\omega_{N}} \int_{\Gamma}\left(\frac{(x-y)}{|x-y|^{N}}\right) \psi(y) d \sigma(y) .
\end{aligned}
$$


As it can be seen from the results in [6] Ch. 3, the restrictions $\left(u_{ \pm}, p_{ \pm}\right)$of

$$
(V(\cdot, h-\llbracket \tau(w+v, q) \rrbracket n), Q(\cdot, h-\llbracket \tau(w+v, q) \rrbracket n))
$$

to $\Omega_{+}$and $\mathbb{R}^{N} \backslash \bar{\Omega}_{+}$, respectively, solve (A.19) in a classical sense, provided $h-\llbracket \tau(w+v, q) \rrbracket n$ is continuous. (Observe that precise regularity properties of $\left(u_{ \pm}, p_{ \pm}\right)$can be obtained from the fact that the mapping $\left.\psi \mapsto V(\cdot, \psi)\right|_{\partial \Omega_{+}}$is a pseudodifferential operator of order -1 as well as from regularity theory for the Stokes-Dirichlet problem, cf. Section 3.3, 3.5 in [6].)

Since $u_{+}$is divergence free, it follows that $\int_{\partial \Omega_{+}} u_{+} \cdot n=0$, and $\llbracket u \rrbracket=0$ on $\partial \Omega_{+}$implies $\int_{\partial \Omega_{+}} u_{-} \cdot n=0$. Hence, since also $u_{-}$is divergence free, it follows that $\int_{\partial C} u_{-} \cdot n_{\partial C}=0$. Thus, $\int_{\partial C}\left(u_{-}+v_{-}\right) \cdot n_{\partial C}=0$.

Let $(\Phi, P)$ a (smooth across $\left.\partial \Omega_{+}\right)$solution of the following Dirichlet problem for the Stokes equations (which exists since $\int_{\partial C}\left(u_{-}+v_{-}\right) \cdot n_{\partial C}=0$, cf. [6, Ch. 3]):

$$
\begin{aligned}
-v \Delta \Phi+\nabla P & =0 & & \text { in } C, \\
\operatorname{div} \Phi & =0 & & \text { in } C, \\
\Phi & =-\left(u_{-}+v_{-}+w_{-}\right) & & \text {on } \partial C .
\end{aligned}
$$

Summarizing, the pair

$$
\left(w_{ \pm}+v_{ \pm}+u_{ \pm}+\Phi, q_{ \pm}+p_{ \pm}+P\right)
$$

is easily seen to solve (A.1) (with $v_{+}=v_{-}=v$ ) in a classical sense. Therefore, the necessary solvability demand (A.12) is also sufficient. Hence, the range of $\Lambda_{v, v}$ is of codimension 1. Since we know from Section A.1.1 that the kernel of $\Lambda_{v, v}$ is one dimensional, this operator has index 0 . Consequently ( $v>0$ was arbitrary), by homotopic stability of the index, all members of the family $\left\{\Lambda_{v_{+},(1-t) v_{+}+t v_{-}} ; t \in[0,1]\right\}$ have index 0 , in particular this holds for $\Lambda_{v_{+}, v_{-}}$. Since this operator has a one dimensional kernel as well, the following Theorem A.1 can be deduced from the general theory of elliptic boundary value problems (cf. [16, Ch. 4], [19, Theorem 9.32]). In order to economize notation we introduce the quotient spaces $\tilde{\mathcal{F}}:=\mathfrak{F} / \sim_{c}$, where $\mathcal{F} \in\left\{W_{p}^{\alpha}, C^{\alpha}, c^{\alpha}\right\}$ and $\sim_{c}$ is the equivalence relation introduced in Section A.1.1. Here, $C^{\alpha}$ stands for the usual Hölder space and $c^{\alpha}$ denotes the little Hölder space, that is the closure of the smooth functions in $C^{\alpha}$.

Theorem A.1 Let $(r, \beta, p, k) \in(0,1] \times(0,1) \times[1, \infty) \times(\mathbb{N} \cup\{0\})$ satisfy $k+r>1 / p, r \neq 1 / p$ and let $\mathrm{C} \in\{c, C\}$. Suppose that $\left(f_{ \pm}, g_{ \pm}, h, l\right)$ of class

(i) $W:=\left[W_{p}^{k+r-1}\left(\Omega_{ \pm}\right)\right]^{N} \times W_{p}^{k+r}\left(\Omega_{ \pm}\right) \times\left[W_{p}^{k+r-1 / p}\left(\partial \Omega_{+}\right)\right]^{N} \times\left[W_{p}^{k+r+1-1 / p}\left(\partial \Omega_{+}\right)\right]^{N}$ or

(ii) $\mathrm{C}:=\left[\mathrm{C}^{k+\beta}\left(\bar{\Omega}_{ \pm}\right)\right]^{N} \times \mathrm{C}^{k+1+\beta}\left(\bar{\Omega}_{ \pm}\right) \times\left[\mathrm{C}^{k+1+\beta}\left(\partial \Omega_{+}\right)\right]^{N} \times\left[\mathrm{C}^{k+2+\beta}\left(\partial \Omega_{+}\right)\right]^{N}$ satisfies $\int_{\Omega_{+}} g_{+}+\int_{\Omega_{-}} g_{-}=-\int_{\partial \Omega_{+}} l \cdot n$. In both cases problem (A.1) possesses a solution $\left(u_{ \pm}, p_{ \pm}\right)$which is unique up to an additive constant for $p_{ \pm}$. This constant is the same in both phases. In case i) $\left(u_{ \pm}, p_{ \pm}\right)$belongs to the class $\left[W_{p}^{k+r+1}\left(\Omega_{ \pm}\right)\right]^{N} \times W_{p}^{k+r}\left(\Omega_{ \pm}\right)$and satisfies the a priori estimate

$$
\left\|\left(u_{ \pm},\left[p_{ \pm}\right]\right)\right\|_{\left[W_{p}^{k+r+1}\left(\Omega_{ \pm}\right)\right]^{N} \times \tilde{W}_{p}^{k+r}\left(\Omega_{ \pm}\right)} \leqslant \gamma\left\|\left(f_{ \pm}, g_{ \pm}, h, l\right)\right\|_{W}
$$

with a positive constant $\gamma$ independent of $\left(f_{ \pm}, g_{ \pm}, h, l\right)$. In case ii) $\left(u_{ \pm}, p_{ \pm}\right)$belongs to the class $\left[\mathrm{C}^{k+2+\beta}\left(\bar{\Omega}_{ \pm}\right)\right]^{N} \times \mathrm{e}^{k+1+\beta}\left(\bar{\Omega}_{ \pm}\right)$and satisfies the estimate

$$
\left\|\left(u_{ \pm},\left[p_{ \pm}\right]\right)\right\|_{\left[\mathrm{C}^{k+2+\beta}\left(\bar{\Omega}_{ \pm}\right)\right]^{N} \times \tilde{\mathrm{e}}^{k+1+\beta}\left(\bar{\Omega}_{ \pm}\right)} \leqslant \gamma\left\|\left(f_{ \pm}, g_{ \pm}, h, l\right)\right\|_{\mathbf{C}}
$$


with a positive constant $\gamma$ independent of $\left(f_{ \pm}, g_{ \pm}, h, l\right)$. The symbol $\left[p_{ \pm}\right]$stands for the class $\left\{p_{ \pm}+\zeta ; \zeta \in \mathbb{R}\right\}$.

For a given domain $\bar{\Omega}_{+} \subset C$ with smooth boundary we denote by $\Lambda_{\Omega_{ \pm}}$the operator defined in (A.13). Using this notation (and those defined in Section 4), we are able to work out that the solution operator $s(\rho)$ of the problem (4.3) depends smoothly on $\rho$.

Corollary A.2 There is a neighbourhood $U$ of 0 in $W_{p}^{3-3 / p}(S)$ such that $s(\rho)$ is well defined for all $\rho \in U \cap$ Ad and $s \in C^{\infty}\left(U \cap A d,\left[W_{p}^{2-2 / p}\left(D_{ \pm}\right)\right]^{N}\right)$. Moreover, if $\delta \geqslant 0$ is given, then there is a neighbourhood $V$ of 0 in $\mathbb{E}(\delta)$ such that $\tilde{s}$ given by

$$
\tilde{s}\left(\left(\mu_{ \pm}, \sigma\right)\right)(t):=\left(0,0,\left.s(\sigma(t))\right|_{S} \cdot n_{S}\right)
$$

is well defined for all $\left(\mu_{ \pm}, \sigma\right) \in V$ and $\tilde{s} \in C^{\infty}\left(V, L_{p}\left(\mathbb{R}^{+}, E_{0}\right)\right)$.

Proof. Let $\Lambda(\rho):=\theta_{\rho}^{*} \Lambda_{\left(\Omega_{\rho}\right)_{ \pm}} \theta_{*}^{\rho}$. Carrying out the transformation of the differential operators involved in (4.3) (cf. the proofs of Theorem 3.1 in [9], Lemma 4.2 in [7]) we obtain

$$
s(\rho)=P \Lambda(\rho)^{-1}(0,0, H(\rho) n(\rho), 0),
$$

where $P\left(u_{+}, p_{+}, u_{-}, p_{-}\right):=u$ and $\Lambda, H$ and $n$ depend all smoothly on $\rho$. Since $\Lambda(0)$ is precisely the operator considered in Theorem A.1 (with $\Omega_{ \pm}:=D_{ \pm}$), the first assertion is obtained by standard perturbation arguments for isomorphisms.

Observe that due to the embedding (4.7) we may assume that $\sigma\left[\mathbb{R}^{+}\right] \subset \operatorname{Ad} \cap W_{p}^{3-3 / p}(S)$ provided $\left\|\left(\mu_{ \pm}, \sigma\right)\right\|_{\mathbb{E}(\delta)}$ is small enough. Hence the second assertion is a consequence of mapping and smoothness properties of concerning Nemytskij operators, cf. [15].

\section{A.2 A few functional analytic tools}

Lemma A.3 Let $H, V$ be complex vector spaces and $A: D(A) \subset H \rightarrow H$ be a linear operator. Let $(\cdot \mid \cdot)$ be a sesquilinear form on $D(A) \times D(A), \Phi \in \mathcal{L}(H, V)$ and

$$
\mathcal{O}:=\{z \in D(A) ;(z \mid h)=0 \text { for all } h \in D(A)\} .
$$

Assume that

(i) $(A u \mid u)=0$ iff $u \in \eta(A)$;

(ii) $\mathbb{R}(A) \subset \eta(\Phi)$;

(iii) $\eta(\Phi) \cap \eta(A) \subset \mathcal{O}$;

Then $\mathbb{R}(A) \cap \eta(A)=\{0\}$.

Proof. Let $z \in \mathbb{R}(A) \cap \eta(A), z=A y$ for some $y \in D(A)$. Then (because of ii), iii) ) $z \in \mathcal{O}$ and

$$
0=(z \mid y)=(A y \mid y),
$$

and hence, because of i), $y \in \eta(A)$ i.e. $z=A y=0$.

Lemma A.4 Let $H$ be a complex vector space, $A: D(A) \subset H \rightarrow H$ and let $(\cdot \cdot \cdot)$ be a sesquilinear form on $D(A) \times D(A)$. Assume additionally that

(i) $(A u \mid u) \in[0, \infty)$ for all $u \in D(A)$; 
(ii) $(u \mid u) \in[0, \infty)$ for all eigenvectors $u$ of $A$;

(iii) if $(u \mid u)=0$ for some eigenvector $u$ of $A$, then $u \in \eta(A)$.

Then all eigenvalues of $A$ are nonnegative.

Proof. Let $\lambda \neq 0$ be a (possibly complex) eigenvalue of $A$. Then, if $u \in D(A)$ is a corresponding eigenvector, we have by ii) and iii) that $(u \mid u)>0$. The assertion follows from $\lambda(u \mid u)=(\lambda u \mid u)=$ $(A u \mid u) \geqslant 0$.

Lemma A.5 Let $X$ be a Banach space, $N$, R linear subspaces with the properties that $N \cap R=\{0\}$, $\operatorname{dim}(N)=\operatorname{codim}(R)=M<\infty$ and that $R$ is closed. Then the quotient map $Q: X \rightarrow X / R$, $y \mapsto y+R$ induces a topological isomorphism from $N$ onto $X / R$ and it vanishes on $R$. Moreover, $X=N \oplus R$ algebraically and topologically.

Proof. It is straightforward to check that $\left.Q\right|_{N}$ is a topological isomorphism onto the finite dimensional Banach space $X / R$ and that $Q$ vanishes on $R$. Moreover, $P:=\left(\left.Q\right|_{N}\right)^{-1} \circ Q$ is a continuous projection of $X$ onto $N$, hence

$$
X=N \oplus \eta(P), \quad \operatorname{dim}(X / \eta(P))=M .
$$

Since $R \subset \eta(P)$ and $\operatorname{dim}(X / R)=M$, we find $\operatorname{dim}(\eta(P) / R)=\operatorname{dim}(X / R)-\operatorname{dim}(X / \eta(P))=0$ and thus $\eta(P)=R$.

Since the range of a Fredholm operator is always closed, we get the following

Corollary A.6 If $X, Y$ are Banach spaces such that $Y \subset X$, and if $F \in \mathcal{L}(Y, X)$ is a Fredholm operator of index 0 that satisfies $\mathbb{R}(F) \cap \eta(F)=\{0\}$, then $X=\eta(F) \oplus \mathbb{R}(F)$ algebraically and topologically. Moreover, $P:=\left(Q \ln _{(F)}\right)^{-1} \circ Q$ is a continuous projection of $X$ onto $n(F)$.

\section{References}

1. Brezzi, F. \& Fortin, M., Mixed and Hybrid Finite Element Methods, Springer, New York 1991. Zb10788. 73002 MR1115205

2. Denk, R., Prüß, J. \& Zacher, R., Maximal $L_{p}$-regularity of parabolic problems with boundary dynamics of relaxation type, Journ. Funct. Anal. 255 (2008), 3149-3187. Zbl1160. 35030 MR2464573

3. Escher, J., Classical solutions to a moving boundary problem for an elliptic-parabolic system, Interfaces and free boundaries, 6 (2004), 175-193. Zbl1057.35097 MR2079602

4. Escher, J. \& Simonett, G., A center manifold analysis for the Mullins-Sekerka model, J. Differential Equations 143 (1998), 267-292. Zb10896.35142 MR1607952

5. Kato, T., Perturbation Theory for Linear Operators, Springer, Berlin, 1966. Zb10148.12601 MR0203473

6. Ladyzhenskaya, O., The Mathematical Theory of Viscous Incompressible Flow, Gordon and Breach, New York 1963. Zbl0121.42701 MR0155093

7. Lippoth, F. \& Prokert, G., Classical solutions for a one phase osmosis model, Journal of Evolution Equations 12 (2012), 413-434. Zbl1254.35250 MR2923941

8. Lippoth, F. \& Prokert, G., Stability of equilibria for a two-phase osmosis model, NoDEA Nonlinear Diff. Eq. Appl. 21 (2014), 129-149. Zbl1301. 35223 MR3158804

9. Lippoth, F., Peletier, M. \& Prokert, G., A moving boundary problem for the Stokes equations involving osmosis: Variational modelling and short-time well-posedness. European J. Appl. Math. 27 (2016), 647666. MR3517540

10. Meurs, P.J.P. v.: Osmotic cell swelling in the fast diffusion limit, MSc thesis, Eindhoven University of Technology 2011, http://alexandria.tue.nl/extra1/afstversl/wsk-i/meurs2011.pdf. 
11. Pickard, W.F., Modelling the Swelling Assay for Aquaporin Expression, J. Math. Biol. 57 (2008), 883903. Zbl1161. 92023 MR2439677

12. Prüß, J., Simonett, G. \& Zacher, R., Qualitative behaviour of solutions for thermodynamically consistent Stefan problems with surface tension, Arch. Ration. Mech. Anal. 207 (2013), 611-667. Zbl1269. 80004 MR3005325

13. Prüß, J., Simonett, G. \& Zacher, R., On normal stability for nonlinear parabolic equations, Discrete Contin. Dyn. Syst. 2009, Dynamical Systems, Differential Equations and Applications, 7th AIMS Conference, suppl., 612-621. Zb11194.35047 MR2648185

14. Rubinstein, L. \& Martuzans, B., Free Boundary Problems Related to Osmotic Mass Transfer Through Semipermeable Membranes, Gakkotosho, Tokyo 1995.

15. Runst, T. \& Sickel, S., Sobolev Spaces of Fractional Order, Nemitskij Operators, and Non-linear Partial Differential Equations, de Gruyter Series in Nonlinear Analysis and Applications, New York, 1996. Zb10873.35001 MR1419319

16. Triebel, H., Theory of Function Spaces, Birkhäuser, Basel, 1983.

17. Verkman, A. S., Water channels in cell membranes, Annu. Rev. Physiol. 54 (1992), 97-108.

18. Verkman, A. S., Solute and makromolecular diffusion in cellular aqueous compartments, Trends Biochem. Sci. 23 (2000), 27-33.

19. Wloka, J. T., Rowley, B. \& Lawruk, B., Boundary Value problems for Elliptic Systems. Cambridge University Press 1995. Zb10836.35042 MR1343490

20. Zaal, M. M., Linear Stability of Osmotic Cell Swelling, MSc thesis, Vrije Universiteit Amsterdam, 2008. http://www.few.vu.nl/ mzl400/bin/scriptie.pdf.

21. Zaal, M. M., Cell Swelling by Osmosis: a Variational Approach, Interfaces and Free Boundaries 14 (2012), 487-520. Zbl1263.35220 MR3016469 